\title{
CDISC SDTM Intervention Model Terminology
}

National Cancer Institute

\section{Source}

National Cancer Institute. CDISC SDTM Intervention Model Terminology. NCI Thesaurus.

Code C99076.

Terminology associated with the intervention model codelist of the Clinical Data

Interchange Standards Consortium (CDISC) Study Data Tabulation Model (SDT M). 